\title{
Ausflug der Forschung: Einmal Lehre und zurüick
}

\section{Tobias Morat}

Gute Hochschullehre sollte eine Einheit von Forschung und Lehre verfolgen (Healy \& Jenkins, 2008; Huber, 2009), damit Studierende gut und breit für potenzielle Berufsfelder qualifiziert werden (Strüder, 2015). Dies bietet zahlreiche Anknüpfungspunkte für innovative Lehr- und Lernkonzepte, besonders beim „Forschenden Lernen“, das sich dadurch auszeichnet,

„dass die Lernenden den Prozess eines Forschungsvorhabens, das auf die Gewinnung von auch für Dritte interessanten Erkenntnissen gerichtet ist, in seinen wesentlichen Phasen [...] (mit)gestalten, erfahren und reflektieren" (Huber, Hellmer \& Schneider, 2009, S. 11).

Dabei kann Forschendes Lernen das Forschungsinteresse von Studierenden erhöhen und die Selbstwirksamkeit der Lernenden steigern (Deicke, Gess \& Rueß, 2014). Es wird allerdings häufig übersehen, dass nicht alle Lernenden zu Forschenden ausgebildet werden sollen und das Forschende Lernen die Entwicklung wichtiger Kompetenzen für jeden Lernenden beinhaltet (Huber, Hellmer \& Schneider, 2009). Besonders die Förderung einer individuellen Lern- und Reflexionsfähigkeit ermöglicht den Studierenden die Entwicklung zu intellektuellen und weltoffenen Persönlichkeiten (Strüder, 2015).

Das Modul „SBG 7 - Intervention“wird in Form eines wissenschaftlichen Projekts im Masterstudiengang „Sport- und Bewegungsgerontologie“(M. Sc. SBG) als Pflicht- 
modul mit sechs Semesterwochenstunden und neun ECTS-Punkten (270 Stunden Arbeitsaufwand) an der Deutschen Sporthochschule Köln (DSHS) durchgeführt. Hierbei werden aktuelle Ergebnisse aus der sportwissenschaftlichen, medizinischen und sportgerontologischen Forschung genutzt, um adäquate Messmethoden und Interventionen im Rahmen eines geeigneten Studiendesigns zu implementieren. Den Lernenden werden im gesamten Modul selbstständige "Strategien“ ermöglicht. Dabei werden Irrtümer und Umwege einerseits in Kauf genommen, andererseits aber auch Erfolge und "fruchtbare Momente" erlebt. Während des gesamten Prozesses besteht darüber hinaus der Anspruch, wissenschaftlich korrektes Arbeiten durch Interaktion, Supervision und Unterstützung des Lehrenden umzusetzen. Auch wenn die Selbstständigkeit der Lernenden im Mittelpunkt des Moduls steht (Huber, Hellmer \& Schneider, 2009), ist es von hoher Bedeutung, dass auch die Lehrenden mitforschen, Fragen haben und neugierig sind. Dies wird in SBG 7 jedes Jahr durch ein innovatives Forschungsvorhaben umgesetzt, dessen Ausgang für alle Beteiligte bis zum Ende offen ist. Die Prüfungsleistung am Ende des Semesters umfasst eine Hausarbeit, im Format eines wissenschaftlichen Zeitschriftenartikels.

\section{$1 \quad$ Lernziele}

\subsection{Inhalte}

Eine Besonderheit des Moduls ist, dass die Lernenden mit ihrer späteren Zielgruppe „ältere Menschen“ (über 65 Jahre) in der Realität umgehen. Das Modul besteht aus einem Seminar (zwei Semesterwochenstunden, SWS) und einer Übung (vier SWS). Die folgenden inhaltlichen Lernziele werden im Laufe des Semesters verfolgt:

(A) Die Studierenden können den aktuellen Forschungsstand in Bezug auf die Zielgruppe bewerten.

(B) Die Studierenden können geeignete Messmethoden zur Erfassung relevanter körperlicher Parameter einschätzen und durchführen.

(C) Die Studierenden können eine wissenschaftliche Studie kreieren (inklusive Planung, Durchführung, Datenauswertung, Evaluation).

(D) Die Studierenden können einen wissenschaftlichen Zeitschriftenartikel über ihre durchgeführte Studie anfertigen. 
Zum Erreichen der vier inhaltlichen Lernziele werden 20 Arbeitspakete (siehe Abb. 1) definiert, welche von den Lernenden im Laufe des Semesters in Interaktion mit der Lehrkraft bearbeitet werden.
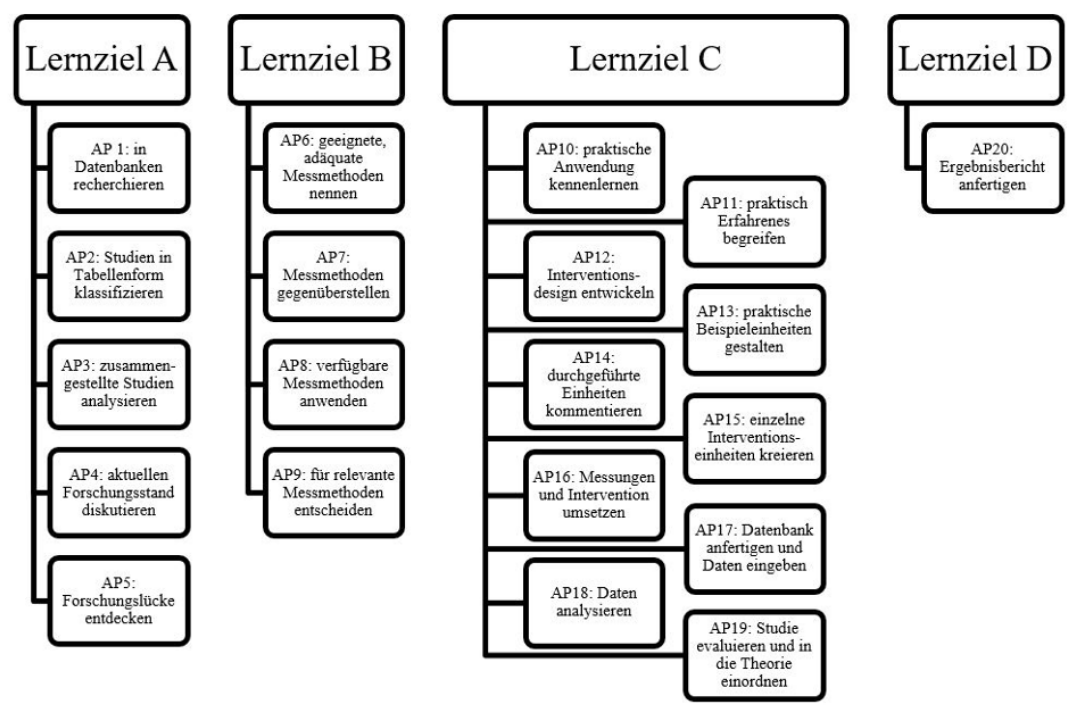

Abb. 1 Zuordnung der 20 Arbeitspakete (= AP) bezogen auf die vier Lernziele

\subsection{Kompetenzen}

Während der Umsetzung mit der Zielgruppe älterer Menschen wird den Lernenden eine vorher nicht bestimmbare Situation geboten, in der sie ihr bis dahin erlerntes Wissen zur Problemlösung verantwortungsvoll und selbstständig einsetzen sollen (Euler, 2005; Huber, Hellmer \& Schneider, 2009; Reiber, 2006). Insbesondere Fähigkeiten, die über das erforderliche Fachwissen und das methodische Können hinausgehen, sind entscheidend, um später anstehende Aufgaben und soziale Konstellationen in der Berufswelt zu bewältigen (Huber, Hellmer \& Schneider, 2009; Pasternack, 2008; Tremp, 2015).

Im Lehr-Lern-Projekt können die Lernenden in den einzelnen Phasen folgende Kompetenzen im Sinne des Forschenden Lernens (Huber, Hellmer \& Schneider, 2009; Wissenschaftsrat, 2000) erwerben bzw. vertiefen: 
- Planung (Teamfähigkeit, Methoden-, Organisations-, Präsentationskompetenz): Auswahl von altersspezifischen Mess- und Trainingsmethoden, adäquate Planung des Studiendesigns wissenschaftlicher Studien mit älteren Menschen;

- Durchführung (Methoden-, didaktische, Vermittlungs-, Trainer-, Kommunikationskompetenz): Testleitungs- (wissenschaftliche Messmethoden in Testungen mit älteren Probandinnen und Probanden anwenden) und Übungsleitungs-Situationen erleben (Training für ältere Menschen anleiten, begleitende Belastungskontrolle und -steuerung des Trainings mit der Zielgruppe durchführen);

- Evaluation (wissenschaftliche Kompetenz, theoriegeleitetes Arbeiten): begleitende Datenerfassung und statistische Auswertungen durchführen, wissenschaftliches Schreiben, Begutachtung wissenschaftlicher Texte, Effektivitätsprüfung von Interventionsprogrammen für ältere Menschen.

\subsection{Berufsorientierung}

Durch die Integration aller Aspekte von Zielsetzung, Umsetzung und Kontrolle bis zur rückblickenden Bewertung im Interventions-Modul werden die Lernenden optimal auf die Anforderungen der späteren Berufswelt vorbereitet. Dabei geht es zum Beispiel darum, bestehende Trainingsprogramme für ältere Menschen zu beurteilen oder neue evidenzbasierte Ansätze für diese Zielgruppe zu entwickeln, erproben und (wissenschaftlich) zu begleiten (North \& Güldenberg, 2008; Reinmann, 2009).

Zu den möglichen Beschäftigungsfeldern der Absolventinnen und Absolventen gehören zum Beispiel die Forschung und die Lehre an Hochschulen, die Mitarbeit in Unternehmen, die spezifische alternsbezogene Produkte und Dienstleistungen anbieten, die betriebliche Gesundheitsförderung, die Aus- und Weiterbildung von Übungsleitungs- und Pflegepersonal, die Bewertung und Qualitätsüberprüfung bestehender Programme in Institutionen oder eine Tätigkeit als Fach- und Führungskraft in Altenhilfeeinrichtungen und Reha-Kliniken.

\section{Ablauf}

In der ersten Semesterphase wird jeweils eine gemeinsame thematische Forschungsfrage entwickelt, die sich unter den Rahmenbedingungen des Modulhandbuchs in einer Studie umsetzen lässt. Nach der Planung der Studie folgt das Herzstück des Moduls, eine mehrwöchige Intervention mit den älteren Personen (inklusive Eingangs- und Ausgangsmessungen vor und nach der Trainingsphase), bevor alle 
erhobenen Daten ausgewertet und in Form eines wissenschaftlichen Artikels verschriftlicht werden. Parallel werden in den Theorieeinheiten die Inhalte und die Struktur des Abschlussberichts in Form eines wissenschaftlichen Zeitschriftenartikels in Kleingruppen erarbeitet und diskutiert; zudem wird ein Schema für die spätere Begutachtung des Artikels erstellt. Nach Fertigstellung der Artikel erfolgt die Begutachtung in zwei Schritten. Zunächst werden die Artikel der Lernenden anonymisiert und per Zufall für die Gutachten neu an die Lernenden verteilt. Durch diese gegenseitige Beurteilung setzen sich die Lernenden auch mit fremden Ergebnissen auseinander, was die Einschätzung der eigenen Leistung objektiviert und die Lernenden voneinander lernen lässt (Huber et al., 2014). Die Lernenden erhalten anschließend (ebenfalls anonym) ein Gutachten zu ihrem Artikel. Anhand des Gutachtens können sie die Qualität ihres Schriftstücks vor der offiziellen Abgabe und Benotung durch die Lehrenden noch verbessern. Mit dem hier vorgestellten Lehr-Lern-Konzept kann im Modul ein vollständiger „Learning Cycle“ im Format des Forschungsprozesses erfolgreich umgesetzt werden (Schneider \& Wildt, 2009).

\section{$3 \quad$ Methodik/Didaktik}

Während des Semesters wird besonders auf den systematischen Wechsel von kollektiven Phasen der Wissensaufnahme (z. B. Vermittlung durch den Lehrenden) und der individuellen Wissensverarbeitung (Erarbeitung relevanter Aspekte in Kleingruppen) geachtet (Sandwich-Prinzip; Reumann et al., 2006; Wahl, 2005). Die beiden Phasen wechseln sich in einer Einheit mehrfach ab und führen nach einer abschließenden Diskussion zu einem gemeinsamen Ergebnis am Ende der Einheit.

Im Rahmen des Lehr-Lern-Konzepts werden neben herkömmlichen Methoden (Gruppenarbeiten, Präsentationen, Diskussionen) auch verschiedene innovative (Medien-gestützte) Methoden wie zum Beispiel 3-Minutes-Talks (3MT) mit Hashtag(\#)-Zusammenfassung oder ein E-Portfolio eingesetzt. Im 3MT haben die Lernenden die Aufgabe, die anderen im Kurs in drei Minuten von ihrer Forschungsfrage und Studienidee, die sie auf Basis ihrer Literaturrecherche entwickelt haben, zu überzeugen. Währenddessen schreiben die anderen Lernenden jeweils drei Hashtag-Begriffe pro 3MT auf. Diese werden im Anschluss ausgezählt und dienen dem Finden eines gemeinsamen Themas für die anschließende Studie. Die Dokumentation des Modulverlaufs erfolgt mittels E-Portfolio, in welchem die Lernenden in Schrift und Bild (Fotos, Videos) Arbeitsverläufe und Zwischenergebnisse darstellen sollen. Durch das E-Portfolio wird von den Lernenden eine nachvollziehbare, konsistente und widerspruchsfreihe Darstellung gefordert, 
sodass sie sich dadurch in Selbstständigkeit üben können (Reinmann, 2009). Im E-Portfolio spielen gewonnene Erkenntnisse, offene Fragen, im Prozess entwickelte und bewährte Kompetenzen, gewählte Lernstrategien und die Beurteilung des Lernfortschritts in Bezug auf die Lernziele eine wichtige Rolle (Arnold et al., 2011; Brunner, Häcker \& Winter, 2006; Deicke, Gess \& Rueß, 2014; Huber, 2008; Kergel \& Heidkamp, 2015; Reis \& Ruschin, 2008).

\section{$4 \quad$ Evaluation und kritische Reflexion}

Die studentische Evaluation am Ende des Semesters zeigte ein sehr positives Bild des Interventions-Moduls. Insbesondere bei den Fragen zum Erwerb von Kenntnissen und Kompetenzen in der Veranstaltung ( $1=$ trifft völlig zu; $4=$ trifft gar nicht $\mathrm{zu})$ wurden der Ausbau fachspezifischer theoretischer Kenntnisse ( $M=1.4 ; S D=0.6)$, Kenntnisse, die für das spätere Berufsleben nützlich sind $(\mathrm{M}=1.7, \mathrm{SD}=0.6)$, fächerübergreifendes Denken und Wissen $(M=1.6$; $S D=0.6)$, die Fähigkeit, Ergebnisse strukturiert zu präsentieren, $(\mathrm{M}=1.7, \mathrm{SD}=0.7)$ und die Fähigkeit, Informationen selbstständig zu erschließen, $(\mathrm{M}=1.5, \mathrm{SD}=0.6)$ durchweg positiv beurteilt. Zusammenfassend wurde das Modul von den Lernenden $(\mathrm{N}=19)$ im Durchschnitt mit $8.4(\mathrm{SD}=0.8)$ bewertet (wobei $1=$ unzureichend und $10=$ ausgezeichnet bedeutet).

Im Rahmen der offenen Rückmeldungen wurden die Struktur und Organisation des Moduls, die klaren Arbeitsaufgaben, die Möglichkeit, das Thema mitzubestimmen, das selbstständige Durchführen der Studie, die Mediennutzung, der hohe Praxisanteil, die vielfältigen Arbeitsformen und die flexible Betreuung durch die Lehrkraft mehrfach als besonders positiv hervorgehoben. Darüber hinaus nahmen die Lernenden das Durchlaufen des Forschungsprozesses von Anfang bis Ende als sehr innovativ und gewinnbringend wahr und betonten diese Gegebenheit als wichtiges Alleinstellungsmerkmal. Allerdings wurden aber auch mehr Unterstützung bei der Datenauswertung, eine bessere Aufgabenverteilung, mehr Austausch mit anderen Arbeitsgruppen und eine längere Bearbeitungszeit für anstehende Aufgaben gewünscht sowie die eigenständige Zeiteinteilung als erschwerend bewertet.

Den Lehrenden kommt in diesem Konzept eine Schlüsselrolle zu. Sie sollten einerseits als Vorbild für den wissenschaftlichen Nachwuchs (die Lernenden) fungieren, dennoch ist auch eine Interaktion auf Augenhöhe für das Forschende Lernen erforderlich. Dieser Spagat stellt eine der Herausforderungen im Lehr-Lern-Konzept dar, mit welchem sich die Lehrenden vorab gründlich auseinandersetzen sollten. Des Weiteren ist die Umsetzung des Lehr-Lern-Konzepts innerhalb der Vorlesungszeit von ca. 15 Semesterwochen sehr ambitioniert und es müssen teilweise aus zeitlichen 
Gründen an einigen Stellen auch pragmatische Entscheidungen getroffen werden - was hinsichtlich der wissenschaftlichen Qualität des Projekts kritisch zu sehen ist. Darüber hinaus steht und fällt das Lehr-Lern-Konzept mit den Kompetenzen, aber insbesondere auch dem Engagement der Lehrenden - und auch der Lernenden. Verfolgen nicht alle gleichermaßen das Gelingen des Gesamtprojekts, ist dessen reibungsloser und erfolgreicher Ablauf bis zum Semesterende in Gefahr. Um mit der Prüfungsleistung noch mehr Aspekte des Lehr-Lern-Konzeptes abzudecken, sollte über eine Anpassung der abschließenden Prüfungsleistung weg von einem wissenschaftlichen Zeitschriftenartikel hin zu einer mehr kompetenzorientierten Form nachgedacht werden.

Dieses Lehr-Lern-Konzept wurde inzwischen auch auf das Bachelorniveau für Profilvertiefungen von zwei Bachelorstudiengängen an der DSHS angepasst und wird auch dort erfolgreich umgesetzt. Es ließe sich auch gut auf andere Fächer und Hochschulen übertragen, da die Vorgehensweisen im Sinne des Forschungszyklus in vielen anderen Disziplinen ähnlich sind. Besonders in Studiengängen mit einem hohen Anteil wissenschaftlicher Inhalte hat es viel Potenzial. Die Kursgröße, die verfügbare Zeit und die Erfahrung der Lehrenden und Lernenden mit einem solchen Lehr-Lern-Konzept sind hierbei wichtige Faktoren. Dieses Konzept bietet speziell für die klassisch anwendungs- bzw. praxisorientierten (Fach-)Hochschulen, im Beispiel eine Sporthochschule, beste Möglichkeiten, den wissenschaftlichen Zugang in der sich zur Wissensgesellschaft verändernden Arbeitswelt in die Lehre zu integrieren (Hube, 2005; Willke, 2001).

\section{$5 \quad$ Projektinfos}

Das vorgestellte Lehr-Lern-Konzept wurde 2014 von der Deutschen Sporthochschule Köln im Programm „Forschungsorientierte Lehre stärken“ gefördert und wurde vom Stifterverband für die Deutsche Wissenschaft mit einem Junior-Fellowship für Innovationen in der Hochschullehre ausgezeichnet (siehe: https://www.stifterverband.org/lehrfellowships/fellowships-hochschullehre-fellows-2014 [09.09.2019]).

Im Rahmen des Moduls (siehe https://www.dshs-koeln.de/studium/studienangebot/master/msc-sport-und-bewegungsgerontologie/ [09.09.2019]) ist auch ein Video über das hier vorgestellte Lehr-Lern-Konzept entstanden (siehe https:// www.youtube.com/watch? $=Y$ YxW1aW_RiE [09.09.2019]). Die Ergebnisse der Studien im Modul „Intervention“ lieferten außerdem Anknüpfungspunkte, die zu einer Fortführung in Form eines größeren Folgeprojekts und einer Masterarbeit führten (Morat et al., 2017). 


\section{Literatur}

Arnold, P., Killian, L., Thillosen, A. \& Zimmer, G. (2011). Handbuch E-Learning: Lehren und Lernen mit digitalen Medien. Bielefeld: Bertelsmann.

Brunner, I., Häcker, T. \& Winter F. (2006). Handbuch Portfolioarbeit. Seelze: Klett, Kallmeyer.

Deicke, W., Gess, C. \& Rueß, J. (2014). Increasing Students' Research Interest through Research-Based Learning. Council on Undergraduate Research Quarterly, 35(1), S.27-33.

Euler, D. (2005). Forschendes Lernen. In S. Spoun \& W. Wunderlich (Hrsg.), Studienziel Persönlichkeit: Beiträge zum Bildungsauftrag der Universität heute (S.253-272). Frankfurt: Campus.

Healy, M. \& Jenkins, A. (2008). Developing Students as Researchers. UC Magazine, S. 17-19.

Hube, G. (2005). Beitrag zur Analyse und Beschreibung der Wissensarbeit. Heimsheim: Jost Jetter.

Huber, L. (2008). 'Kompetenzen' prüfen? In S. Dany, B. Szczyrba \& J. Wildt (Hrsg.), Prüfungen auf die Agenda! Hochschuldidaktische Perspektiven auf Reformen im Prüfungswesen (S. 12-26). Bielefeld: Blickpunkt Hochschuldidaktik.

Huber L. (2009). Warum Forschendes Lernen nötig und möglich ist. In L. Huber, J. Hellmer \& F. Schneider (Hrsg.), Forschendes Lernen im Studium: Aktuelle Konzepte und Erfahrungen (S.9-35). Bielefeld: UVW.

Huber, L., Hellmer, J. \& Schneider, F. (2009). Forschendes Lernen im Studium: Aktuelle Konzepte und Erfahrungen. Bielefeld: UVW.

Huber, L., Pilniok, A., Sethe, R., Szcyrba, B. \& Vogel, M. (2014). Forschendes Lehren im eigenen Fach: Scholarship of Teaching and Learning in Beispielen. Bielefeld: Bertelsmann.

Kergel, D. \& Heidkamp, B. (2015). Forschendes Lernen mit digitalen Medien: Ein Lehrbuch. Münster: Waxmann.

Morat, T., Krueger, J., Gaedtke, A., Preuß, M., Latsch, J. \& Predel, H.-G. (2017). Effects of 12 weeks of Nordic Walking and XCO Walking training on the endurance capacity of older adults. European Review of Aging and Physical Activity. DOI 10.1186/s11556-017-0186-2.

North, K. \& Güldenberg, S. (2008). Produktive Wissensarbeiter(er): Performance messen, Produktivität steigern, Wissensarbeiter entwickeln. Wiesbaden: Gabler.

Pasternack, P. (2008). Teilweise neblig, überwiegend bewölkt: Ein Wetterbericht zur deutschen Hochschulsteuerung. In B. M. Kehm (Hrsg.), Hochschule im Wandel: Die Universität als Forschungsgegenstand (S. 194-206). Frankfurt: Campus.

Reiber, K. (2006). Wissen - Können - Handeln: Ein Kompetenzmodell für lernorientiertes Lehren. (Tübinger Beiträge zur Hochschuldidaktik 2/1). Tübingen.

Reinmann, G. (2009). Wie praktisch ist die Universität? Vom situierten zum Forschenden Lernen mit digitalen Medien. In L. Huber, J. Hellmer \& F. Schneider (Hrsg.), Forschendes Lernen im Studium: Aktuelle Konzepte und Erfahrungen (S.36-52). Bielefeld: UVW.

Reis, O. \& Ruschin, S. (2008). Kompetenzorientiert prüfen - Baustein eines gelungenen Paradigmenwechsels. In S. Dany, B. Szczyrba \& J. Wildt (Hrsg.), Prüfungen auf die Agenda! Hochschuldidaktische Perspektiven auf Reformen im Prüfungswesen (S. 45-57). Bielefeld: Blickpunkt Hochschuldidaktik.

Reumann, M., Mohr, M., Dössel, O. \& Diez, A. (2006). Grundlagenveranstaltungen neu verpackt: Vorlesung, Übung und Tutorien im koordinierten Zusammenspiel. In B. Berendt, H.-P. Voss \& J. Wildt (Hrsg.), Neues Handbuch Hochschullehre: Lehren und Lernen effizient gestalten. Berlin: Raabe. 
Schneider, R. \& Wildt, J. (2009). Forschendes Lernen und Kompetenzentwicklung. In L. Huber, J. Hellmer \& F. Schneider (Hrsg.), Forschendes Lernen im Studium: Aktuelle Konzepte und Erfahrungen (S. 53-69). Bielefeld: UVW.

Strüder, H. (2015). 2015 Hochschulentwicklungsplan. Köln: Deutsche Sporthochschule Köln. Verfügbar unter https://www.dshs-koeln.de/fileadmin/redaktion/Aktuelles/Publikationen_und_Berichte/Publikationen/HEP/Hochschulentwicklungsplan-DSHS.pdf [27.10.2015].

Tremp, P. (2015). Forschungsorientierung und Berufsbezug im Studium. Bielefeld: Bertelsmann. Wahl, D. (2005). Lernumgebungen erfolgreich gestalten: Vom trägen Wissen zum kompetenten Handeln. Bad Heilbrunn: Klinkhardt.

Willke, H. (2001). Systemisches Wissensmanagement. Stuttgart: UTB.

Wissenschaftsrat (2000). Empfehlungen zur Einführung neuer Studienstrukturen und -abschlüsse (Baccalaureus/Bachelor - Magister/Master) in Deutschland. Verfügbar unter https://www.wissenschaftsrat.de/download/archiv/4418-00.pdf [27.10.2015].

\section{Zum Autor}

Dr. Tobias Morat, Sportwissenschaftler, arbeitet am Institut für Bewegungs- und Sportgerontologie der Deutschen Sporthochschule Köln. Er ist dort seit 2009 Wissenschaftlicher Mitarbeiter mit diesen Forschungs-/Arbeitsschwerpunkten: Sarkopenie, Kraft- und Ausdauertraining bei älteren Menschen, Sling-Training, Interventionsforschung, Forschendes Lernen, Studiengangsweiterentwicklung und -koordination. Er ist Studiengangskoordinator des Masterstudienganges „M. Sc. Sport- und Bewegungsgerontologie (SBG)“ (bis WS16/17 als M. A.) und unterrichtet in verschiedenen Veranstaltungen des SBG Masters und in Bachelorveranstaltungen. Tobias Morat ist seit 2014 ein Fellow des Stifterverbands für die deutsche Wissenschaft. E-Mail: t.morat@dshs-koeln.de

Open Access Dieses Kapitel wird unter der Creative Commons Namensnennung 4.0 International Lizenz (http://creativecommons.org/licenses/by/4.0/deed.de) veröffentlicht, welche die Nutzung, Vervielfältigung, Bearbeitung, Verbreitung und Wiedergabe in jeglichem Medium und Format erlaubt, sofern Sie den/die ursprünglichen Autor(en) und die Quelle ordnungsgemäß nennen, einen Link zur Creative Commons Lizenz beifügen und angeben, ob Änderungen vorgenommen wurden.

Die in diesem Kapitel enthaltenen Bilder und sonstiges Drittmaterial unterliegen ebenfalls der genannten Creative Commons Lizenz, sofern sich aus der Abbildungslegende nichts anderes ergibt. Sofern das betreffende Material nicht unter der genannten Creative Commons Lizenz steht und die betreffende Handlung nicht nach gesetzlichen Vorschriften erlaubt ist, ist für die oben aufgeführten Weiterverwendungen des Materials die Einwilligung des jeweiligen Rechteinhabers einzuholen. 